\title{
Design and Evaluation of a Low-Cost Acoustic Chamber for Underwater Networking Experiments.
}

\author{
Francesca Meneghello ${ }^{\S}$, Filippo Campagnaro ${ }^{\S}$, Roee Diamant ${ }^{\S}$, Paolo Casari ${ }^{\sharp}$, Michele Zorzi ${ }^{\S}$ \\ ${ }^{\S}$ Department of Information Engineering, University of Padova, via Gradenigo 6/B, 35131 Padova, Italy \\ ${ }^{\ddagger}$ Department of Marine Technology, University of Haifa, Israel \\ "IMDEA Networks Institute, Madrid, Spain
}

\begin{abstract}
Testing acoustic equipment before sea experiments is a necessary step, which usually requires large and expensive facilities. In this paper, we present the design guidelines, structure and details of a small-scale, low-cost acoustic chamber for in-lab testing of underwater acoustic networks. The chamber has been assembled with the objective to be of low cost and limited size: therefore, its installation fits small university laboratories that cannot afford large testing pools.

The chamber was designed to mitigate the extreme multipath which, in a small chamber, makes communications unreliable. Considering this challenge, our chamber includes a phono-absorbing coating on the walls and floor, to be optionally complemented by a panel of the same coating material, to be installed at the water surface level.

After providing the details of several phono-absorbing materials to motivate our specific choice, we carry out a number of transmission experiment with EvoLogics modems, proving that our design substantially reduces the severe multipath and thereby improves the communications quality.
\end{abstract}

\section{Categories and Subject Descriptors}

B.8.2 [Hardware]: Performance and Reliability-Performance Analysis and Design Aids; C.2.0 [Communication/ Networking and Information Technology]: GeneralData communications

\section{Keywords}

Underwater acoustic networks; laboratory tests; acoustic chamber; phono-absorbing coating design; EvoLogics modems; experiments; packet delivery ratio; multipath evaluation.

\section{INTRODUCTION AND RELATED WORK}

Performing a sea experiment to test underwater acoustic networks (UWANs) requires precise coordination between ${ }^{*}$ This work has been supported in part by the US Office of
Naval Research under Grant no. N62909-14-1-N127.

Permission to make digital or hard copies of part or all of this work for personal or classroom use is granted without fee provided that copies are not made or distributed for profit or commercial advantage and that copies bear this notice and the full citation on the first page. Copyrights for third-party components of this work must be honored. For all other uses, contact the owner/author(s).

WUWNet'16, October 24-26 2016, Shanghai, China

(C) 2016 Copyright held by the owner/author(s).

ACM ISBN 978-1-4503-4637-5/16/10.

DOI: http://dx.doi.org/10.1145/2999504.3001086 the network nodes. Since a failure in such an experiment implies significant costs for re-organizing and executing a fresh field trial, the software should be bug-free, and the hardware should be able to withstand the foreseen sea conditions. This sensitivity to potential failure makes it crucial to perform in-lab tests to validate the software and the equipment before going to the field.

The in-lab testing process involves both simulations and lab experiments. The former is a relatively easy process which is performed using an event-based simulator, e.g., [1-5], whereas the latter involves the same hardware to be employed later in the field trial, and should be regarded as a small-scale experiment, which requires the use of a testing pool or chamber. Although the long propagation delays experienced in real scenarios cannot be reproduced in the latter case, such a setting does push the lab testing conditions one step closer to what will be found in a field trial environment, and makes it possible to test transmissions at, e.g., higher power levels, which would otherwise damage the projectors if employed while the modem is not submerged [6]. However, small test chambers carry a number of challenges among which the most dominant is the strong multipath which exists due to the small-scale testing facility (see Fig. 1). While such a challenging environment may provide a valuable tool for worst-case performance evaluation, often this advantage is overcome by the difficulty of finding a proper configuration that allows sufficient network connectivity for the test to be carried out. As an example, Fig. 2 shows a typical tank configuration where, due to strong multipath, it was not possible to obtain a connected network.

The most realistic in-lab experiments are naturally carried out in large and deep testing pools. These environments provide characteristics more akin to shallow water scenarios, giving the research team more insight as to what to expect in a real environment, and thus making it more ready for the sea experiment. However, large testing pools, such as the one employed by CMRE [7], require a large infrastructure which may not be available to smaller research laboratories, e.g., as typically found in universities. As a result, such laboratories may have to skip the in-lab validation process, or to perform it outside the lab, possibly in harbors and marinas, whose environments are typically characterized by high noise levels and strong reverberation. In turn, the validation may require a lengthy set-up phase to identify the ideal conditions for a controlled validation test, with the understanding that such conditions are then highly subject to change over time due to evolving environmental conditions and noise-generating activities in the nearabouts.

When a large pool is not available, smaller tanks may be 


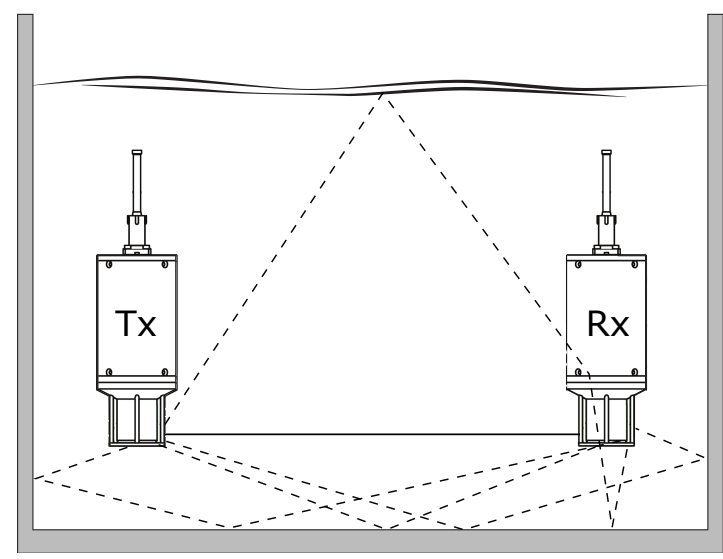

Figure 1: Example of multipath propagation in the tank (dashed) and line-of-sight path (solid).

of interest for making preliminary tests. For example, the authors in [8] performed the tests in a wave flume, which albeit being smaller than a pool, still requires a large facility for its installation. The works described in [9] and [10] were both tested in tanks of different sizes. A different approach is taken in [11], where the authors test acoustic communications in air by employing a specifically constructed emulator, where phono-absorbing material allows to obtain the same attenuation as a $2-\mathrm{km}$ free-space underwater link within a space of $4 \mathrm{~cm}$. The solution does not rely on actual acoustic modems and can thus represent only an intermediate step towards a sea experiment.

In this paper, we tackle the need for a low cost acoustic chamber as a valuable experimentation tool and describe the implementation details of such a chamber built from available low-cost materials. This chamber is now part of an UWAN testbed at the University of Padova, which includes seven EvoLogics S2CR 18/34 modems [12].

Our acoustic chamber is a small 200-liter plastic tank of a rectangular shape of dimensions $67 \mathrm{~cm} \times 57 \mathrm{~cm} \times 52 \mathrm{~cm}$. A picture of the chamber is presented in Fig. 2. The small size of the chamber allows its easy deployment inside the laboratory as well as its displacement for demonstrations. The chamber includes three metal handles to help organize the deployment of acoustic modems at different depth and distances. To reduce the multipath that originates in such a small tank, we use a phono-absorbing material. However, in some cases, there may be a need to preserve some multipath, e.g., when testing the robustness of signal processing and networking protocols in face of adverse channel conditions. Therefore, our design includes a way to control multipath through a removable tank cover.

Our specific contributions are: $i$ ) the systematic analysis of different phono-absorbing materials and of their intank echo reduction effect, including using different levels of tank and water surface coating; ii) a detailed account of our experience with small tank experiments and solutions to make this logistically viable solution also reliable from a communications standpoint; iii) several test results using EvoLogics modems, for which we report both the achieved packet delivery ratio figures and the capability of the phonoabsorbing coating to provide a sufficiently clean signal to the modems' multipath-rejecting physical layer. We remark that the modems work in the $18-34 \mathrm{kHz}$ band, which leads to a

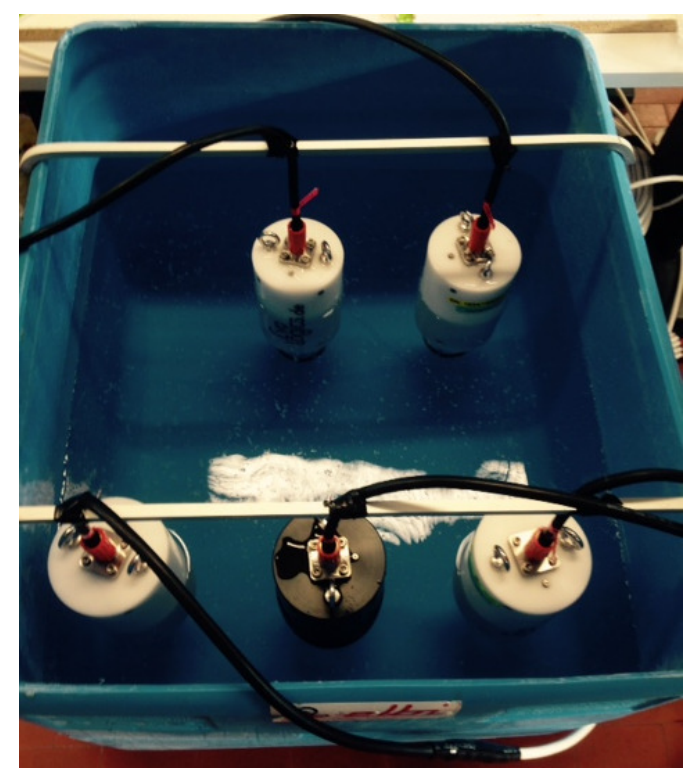

Figure 2: Example of a tank test. In a system with 5 nodes it was not possible to obtain a connected network due to the strong multipath effect.

wavelength of about $5 \mathrm{~cm}$. In these conditions, the chassis of the modems are also turned into significant reflectors.

Our chamber was first employed for testing the handshakebased protocol reported in [13] before a real experiment in the Garda lake, Italy. This protocol exploits the near-far effect and involves power control. The lab testing of this protocol required changing the node's transmission power, changing the position of the nodes, and conveying packets of different sizes to different destinations. To this end, it was essential to overcome the large inter- and intra-symbolinterference caused by the reflections from the boundaries of the tank, a task that was completed successfully thanks to our chamber design.

The remainder of this paper is organized as follows: in Section 2 we discuss the design of the tank and the choice of a phono-absorbing material; in Section 3 we discuss the results of several transmission experiments carried out in the tank, and finally draw our conclusions in Section 4.

\section{DESIGN CONSIDERATIONS FOR THE ACOUSTIC TANK COATING}

To keep the paper self-contained, we start by recalling a few notions on the physical quantities that determine the acoustic behavior of a medium. Every propagation medium is characterized by its density $\rho$ and the speed of sound in the medium, denoted as $c$. Their product $Z=\rho c$ is called acoustic impedance: it can be interpreted as a measure of how hard it is for the sound to propagate in the medium and is measured in Rayls, where 1 Rayl is equivalent to 1 $\mathrm{kg} \cdot \mathrm{m}^{-2} \cdot \mathrm{s}^{-1}$. In general, sound waves propagating through a medium of impedance $Z_{1}=\rho_{1} c_{1}$ and encountering an interface with a second medium of impedance $Z_{2}=\rho_{2} c_{2}$ are partly reflected off the interface and partly refracted inside the second medium. The reflection coefficient that deter- 
mines the fraction of the wave energy reflected back into medium 1 is derived as

$$
R_{12}=\frac{Z_{2} \cos \theta_{i}-Z_{1} \cos \theta_{t}}{Z_{2} \cos \theta_{i}+Z_{1} \cos \theta_{t}},
$$

where $\theta_{i}$ is the angle of incidence of the wavefront on the interface between the first and the second medium, and $\theta_{t}$ is the angle of refraction inside the second medium, both measured with respect to the direction normal to the interface. The coefficient $T_{12}=1-R_{12}$ determines instead the amount of energy transferred to medium 2 . The value $-20 \log _{10} R_{12}$ (in $\mathrm{dB}$ ) is called echo reduction, and conveys the capability of the material to attenuate echos originating from the interface.

Additionally, every propagation medium can absorb the energy of the acoustic wave in different ways, which will be considered in the next subsection. For the moment, assume that there exists a second interface between the second medium and a third medium. The fraction of energy transferred to medium 3 can be computed as $T_{12} T_{23} / A_{2}$, where $A_{2}$ conveys the amount of attenuation incurred by the sound wave as it propagates through medium 2. The value $-20 \log _{10} T_{23}$ (in $\mathrm{dB}$ ), is also called insertion loss, and expresses the capability of medium 2 to retain acoustic energy instead of transmitting it to medium 3.

With reference to the choice of a phono-absorbing coating for our tank in Fig. 2, we remark that there are four propagation media involved: 1) water; 2) the coating material; 3) the tank walls; 4) air. There are therefore three interfaces that acoustic waves interact with. The clear water typically used for in-lab tests has an impedance $Z_{1}=1.48 \mathrm{MRayl}$; the tank walls are made of high-density polyethylene, which has an impedance $Z_{3}=2.3 \mathrm{MRayl}$; finally, air has an impedance $Z_{4}=429$ Rayl. Of course, water and air are in direct contact at the water surface. At this point it becomes clear that in order to achieve a good multipath reduction, we need at least to apply a coating material to the tank walls, and optionally to the water surface/air interface. This material should have the following characteristics: it should be waterproof and exhibit an impedance matching the one of the water and possibly being not too different from the impedance of the tank walls; it should provide good sound absorption properties, high echo reduction and small insertion loss; it should be low cost and possibly off-the-shelf; it should be thin enough not to take up too much space in the tank. In the following, we will survey and discuss the possible choices for the phono-absorbing tank wall coating and present our final choice.

\subsection{Survey of phono-absorbing materials}

Several types of materials can be employed for the wall coating. A first differentiation among them is made based on their density. If its density is less than $1 \mathrm{~g} \cdot \mathrm{cm}^{-3}$, a material is named "expanded:" this class includes open-cell (or porous) materials as well as some classes of closed-cell materials. Otherwise, a material is named "compact."

Porous materials are typically employed for sound dampening in air, as their impedance adapts well to the air's impedance [14]. In this case, sound waves that penetrate the material's pores create small vortices that lead to a quicker energy dissipation. The same property could be employed under water, but the matching between porous materials and water would be much worse, and most of the acoustic energy would be unable to penetrate the material [15]. A different mechanism takes place in the so-called Insulcrete, a mixture of Portland cement and pine wood sawdust: in this case, acoustic energy dissipation originates from the relative motion between the water penetrating the material and the material itself [16,17]. A layer of crushed rocks also behaves as a porous material, that dissipates acoustic energy due to the many reflections that take place in the interstices among the rock fragments [18]. The majority of porous materials are, however, not waterproof, and the penetration of water in the material tends to substantially deteriorate its phonoabsorbing properties.

Several materials absorb acoustic energy by transforming it into heat through elastic deformation. Compact rubbers and high-density polyurethane present this characteristic behavior [15]. Rubbers are specifically important for phonoabsorbency under water, as their acoustic impedance can be adapted to that of water. For the same reason, rubbers work comparatively worse in air, where they are mostly used as insulators $[14,15,19,20]$.

In order to increase phono-absorption, the materials can be physically modified or shaped. For example, porous materials for sound absorption in air are shaped into cones in order to increase the total contact surface and impair the propagation of sound waves. In the case of absorption into water, instead, two approaches are typically employed: $i$ ) filling the material with sawdust, cork dust or similar materials and $i$ ) cavitation of otherwise homogeneous materials. The latter concept (which is more controllable than the former) has been exploited to build the Alberich tiles employed to cover submarines during the second world war [16]. The rubber-based material in [21] exploits the same technique. A specific case of technique $i$ ) above is the addition of metal dust to butyl rubber [17]: this achieves a better matching with the water impedance and increases the attenuation of sound energy in the material. However, this type of rubber tends to absorb water over time, which degrades its phonoabsorbing properties.

The properties of chloroprene rubber (also known by its commercial name neoprene) are studied in [20], where the authors mix the rubber with different quantities of carbon in order to achieve different degrees of hardness, which in turn increases the acoustic impedance of the material while maintaining it around the same order of magnitude of water's acoustic impedance. Moderate proportions of carbon offer an insertion loss less than $2 \mathrm{~dB}$ and an echo reduction in excess of $10 \mathrm{~dB}$, which make the material suitable for phono-absorption applications.

The development of a visco-elastic material of the socalled class of locally-resonant phononic crystals (LRPCs) is described in [22]. These materials are characterized by the insertion of metallic particles of the same size, that act as resonating crystals at the frequencies of interest. The specific material in [22] is based on high- and low-density polyurethane and aluminium foam. The results show high acoustic energy absorption, much higher than achieved by each component alone [14], although the material is prone to a substantial alteration of its acoustic properties in the presence of any defects.

Compact, polyurethane-based tiles that achieve a broadband echo reduction and absorption are presented in [23]. The tiles are well matched to the water impedance, and sound energy absorption is enhanced through the insertion of air micro-spheres and high-density fillers, and by shaping the material in cones. The tiles are sold by Precision 


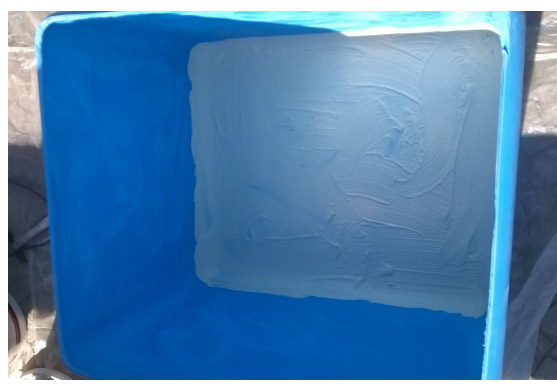

(a)

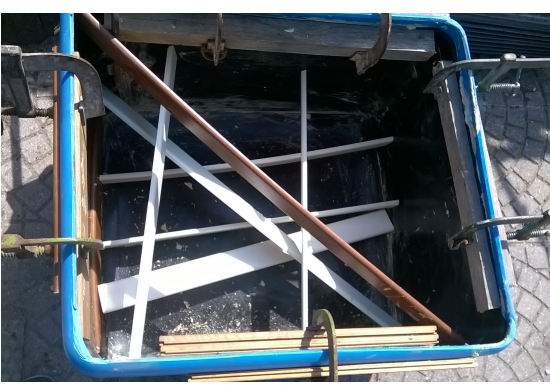

(b)

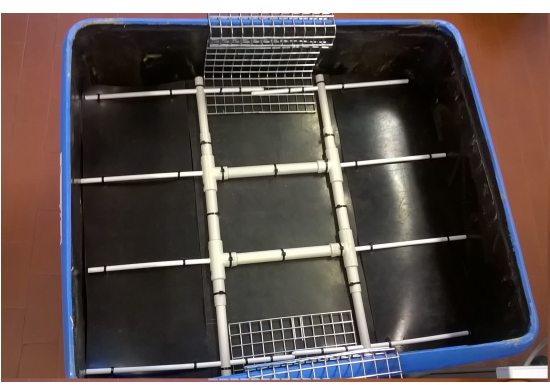

(c)

Figure 3: Different stages of the tank coating process: (a) application of the cement-based glue; (b) application of the neoprene rubber on all sides and drying; (c) finished tank including the surface coating layer.

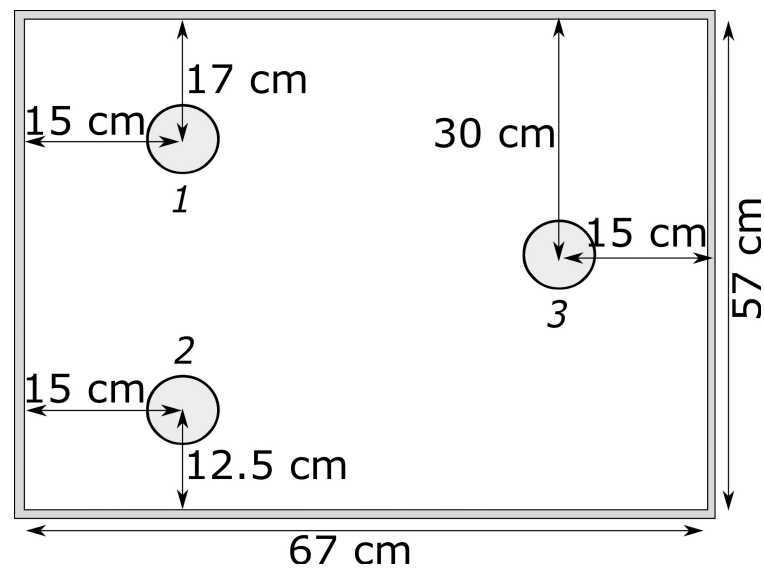

Figure 4: Setting of our experiment: one node at a time transmits one packet per second, while the others measure the multipath.

Acoustics under the commercial name Aptflex SF5048.

A multi-layer material composed of water, marine plywood, butyl rubber loaded with cork dust and aluminium, and butyl rubber cones is presented in [14]. More experiments with the properties of composite materials in the 10$50 \mathrm{kHz}$ range are detailed in [17]. The general conclusion is that the best attenuation is obtained when the rubber is in contact with the water. A summary of the findings of our literature survey on phono-absorbing materials is provided for reference in Tables 1 and 2 .

\subsection{Choice of a phono-absorbing wall coating}

Given the survey and considerations presented in the previous subsection, we finally chose to implement the phonoabsorbing tank wall coating using a 6-mm neoprene rubber layer to be fixed to the tank walls through a two-component cement mortar-based glue of the type employed to insulate pools and terraces. The thickness has been chosen in order to subtract only a minimal amount of space to the tank. The acoustic impedance of the neoprene rubber is $Z_{2}=2.24 \mathrm{MRayl}$, and has the same order of magnitude of water's impedance. We recall that the HDPE tank walls and bottom (impedance $Z_{3}=2.3 \mathrm{MRayl}$ ) are also well matched by the rubber. Other materials have been discarded for several reasons. For instance, Aptflex SF5048 tiles, albeit having a very desirable behavior in the frequency range of interest $(18-34 \mathrm{kHz})$ have been discarded due to their excessive cost. Composite structures such as [14] have also been discarded due to their excessive size, which would leave an insufficient amount of space for the modems inside the tank. More complex materials [15,22] have finally been discarded as they require specific instrumentation to be employed.

We remark that there still exists a highly reflective HDPEair interface, whose impact is now limited thanks to the phono-absorbing characteristics of the neoprene layer. The water surface-air interface is also highly reflective. To compensate for the latter, a structure has been prepared to submerge a planar layer of the same neoprene rubber employed for the tank wall coating. A possible solution for dampening the HDPE-air reflection would be to apply an external layer with high acoustic absorption coefficient and impedance matched to that of HDPE. This solution, however, has not been applied for the moment and has been left as a future extension. Fig. 3 shows different stages of the tank coating process: (a) the application of the cementbased glue; (b) the internal neoprene coating while drying; (c) the final installation including the surface neoprene layer.

\section{EXPERIMENTAL RESULTS}

Our tests have been carried out by submerging three EvoLogics S2CR 18-34 modems in the tank, over different tank coating stages. The position of the modem is as illustrated in Fig. 4, except that the distance between the modems and the perimeter of the walls was reduced with the introduction of the neoprene rubber layer.

The modems allow transmit power control in four levels, corresponding to a source level (SL) of 184, 178, 172 and 164 $\mathrm{dB}$ relative to $1 \mu \mathrm{Pa}$ at $1 \mathrm{~m}$ from the source. For our experiments, we employed a SL of either 164 (SL1) or $178 \mathrm{~dB}$ re $1 \mu \mathrm{Pa}$ at $1 \mathrm{~m}$ (SL2), where the latter is a factor of about 25 greater than the former, in linear scale. With this test, we aim to show that operations with high source levels (possibly used, e.g., in protocols exploiting power control and/or near-far scenarios [13]) can in fact be experimented using our setup, despite the limited space available in the tank.

We are mainly interested in two communication quality measures: packet delivery ratio (PDR), and multipath richness. To measure these quantities, we configure the nodes to transmit 100 packets at a rate of one packet per second. The transmissions take place in turns, so that at any given time one node transmits and the other two nodes attempt receiving the packets and performing multipath measurements.

We start from the PDR values, which are reported in Ta- 
Table 1: General properties of different classes of phono-absorbing materials

\begin{tabular}{lll} 
Type & Advantages & Disadvantages \\
\hline Porous materials & $\begin{array}{l}\text { High attenuation by trapping acoustic } \\
\text { energy in pores. }\end{array}$ & $\begin{array}{l}\text { Acoustic impedance not well matched to water's; } \\
\text { water absorption degrades properties. }\end{array}$ \\
\hline $\begin{array}{l}\text { High-density } \\
\text { visco-elastic materials. }\end{array}$ & $\begin{array}{l}\text { Good water impedance match; limited water } \\
\text { absorption; high attenuation through elastic } \\
\text { deformation. }\end{array}$ & $\begin{array}{l}\text { Poor resistance to hydrocarbons. Other } \\
\text { material-specific disadvantages (see Table 2) }\end{array}$ \\
\hline Composite materials & $\begin{array}{l}\text { Optimally phono-absorbing at the } \\
\text { frequencies of interest for this paper. }\end{array}$ & $\begin{array}{l}\text { Exceedingly thick; typically complicated } \\
\text { preparation and application. }\end{array}$ \\
\hline
\end{tabular}

Table 2: Advantages and disadvantages of specific phono-absorbing materials. For each category, the characteristics listed in Table 1 also apply.

\begin{tabular}{|c|c|c|}
\hline Type & Advantages & Disadvantages \\
\hline \multicolumn{3}{|c|}{ Porous materials } \\
\hline Insulcrete $[14,16,17]$ & $0.9 \leq \rho_{18-34} \leq 0.99$ & $\begin{array}{l}\text { Long and complex preparation; } \\
\text { highly alkaline (requires water } \\
\text { neutralization); tends to form a } \\
\text { thickening surface layer. }\end{array}$ \\
\hline $\begin{array}{l}\text { Crushed rocks (grain } \\
\text { diameter from } 3 \\
\text { to } 5 \mathrm{~mm}[18]\end{array}$ & $0.6 \leq \rho_{18-34} \leq 0.9 ;$ natural resistance to water absorption. & Release impurities in the water. \\
\hline \multicolumn{3}{|c|}{ Visco-elastic materials } \\
\hline Polyurethane $[18,24-27]$ & $\begin{array}{l}0.6 \leq \rho_{18-34} \leq 0.8 ; \text { good water impedance matching; very } \\
\text { limited water absorption; easy to use. }\end{array}$ & $\begin{array}{l}\text { Deteriorates when exposed to } \\
\text { sunlight or to organic solvents. }\end{array}$ \\
\hline $\begin{array}{l}\text { Neoprene } \\
\text { rubber }[19,20,26,28]\end{array}$ & $\begin{array}{l}0.2 \leq \rho_{18-34} \leq 0.6 \text {; good water impedance matching; } \\
\text { adheres to metal and rigid materials; resistant to acids } \\
\text { and bases; good mechanical properties; water-resistant. }\end{array}$ & Poor resistance to hydrocarbons. \\
\hline Butyl rubber $[19,26,28]$ & $\begin{array}{l}0.2 \leq \rho_{18-34} \leq 0.6 ; \text { good water impedance matching; good } \\
\text { resistance to flex, oxidation, gas and sunlight; good } \\
\text { waterproofing and resistance to acids and bases. }\end{array}$ & Not resistant to radiations. \\
\hline Chlorobutyl rubber $[19,26]$ & $\begin{array}{l}\text { Same as butyl rubber plus better resistance to heat and } \\
\text { compression. }\end{array}$ & Not resistant to radiations. \\
\hline Natural rubber $[19,26]$ & $\begin{array}{l}\text { Good water impedance matching; high mechanical energy } \\
\text { loss factor; good resistance to water. }\end{array}$ & $\begin{array}{l}\text { Poor resistance to ozone and } \\
\text { sunlight; } \rho_{18-34} \leq 0.6\end{array}$ \\
\hline $\begin{array}{l}\text { Ethylene-Propylene Diene } \\
\text { Monomer } \\
\text { rubber }[19,26,28]\end{array}$ & $\begin{array}{l}\text { Good water impedance matching; high mechanical energy } \\
\text { loss factor; very good resistance to water, ozone and } \\
\text { sunlight. }\end{array}$ & $\begin{array}{l}\text { Poor resistance to tear; difficult } \\
\text { bonding, especially to metals. }\end{array}$ \\
\hline \multicolumn{3}{|c|}{ Composite materials } \\
\hline $\begin{array}{l}\text { Rubber with sectional } \\
\text { cavities }[21]\end{array}$ & Better sound absorption properties than plain rubber. & $*$ \\
\hline Aptflex SF $5048[23,29]$ & $\begin{array}{l}\text { Specifically designed for tank lining; echo reduction at } \\
\text { least }-30 \mathrm{~dB} \text {. High attenuation in the material. }\end{array}$ & $*$ \\
\hline $\begin{array}{l}\text { Marine plywood }+ \text { cork-Al } \\
\text { powder-loaded butyl } \\
\text { rubber + loaded butyl } \\
\text { rubber wedges }[14]\end{array}$ & Echo reduction at least $-12 \mathrm{~dB}$. & $\begin{array}{l}\text { Optimal performance achieved } \\
\text { only if the water surface is also } \\
\text { covered with the material. }\end{array}$ \\
\hline $\begin{array}{l}\text { Rubber incorporating } \\
\text { materials with high air } \\
\text { content }[15,17,26]\end{array}$ & $*$ & $\begin{array}{l}\text { Absorption of water over time } \\
\text { degrades the material's } \\
\text { properties. }\end{array}$ \\
\hline $\begin{array}{l}\text { Neoprene rubber with } \\
\text { carbon black loading [20] }\end{array}$ & $\begin{array}{l}\text { Improved mechanical properties without compromising } \\
\text { impedance adaptation. Insertion loss less than } 2 \mathrm{~dB} \text {, echo } \\
\text { reduction greater than } 10 \mathrm{~dB} \text {. }\end{array}$ & $*$ \\
\hline $\begin{array}{l}\text { Composition of locally } \\
\text { resonant units of different } \\
\text { sizes }[22,27]\end{array}$ & $\rho_{18-34} \approx 0.9$ & $*$ \\
\hline
\end{tabular}

* indicates that the corresponding characteristics are as indicated in Table 1. 
Table 3: Link packet error rates for several configurations of the tank and of the transmitting modem.

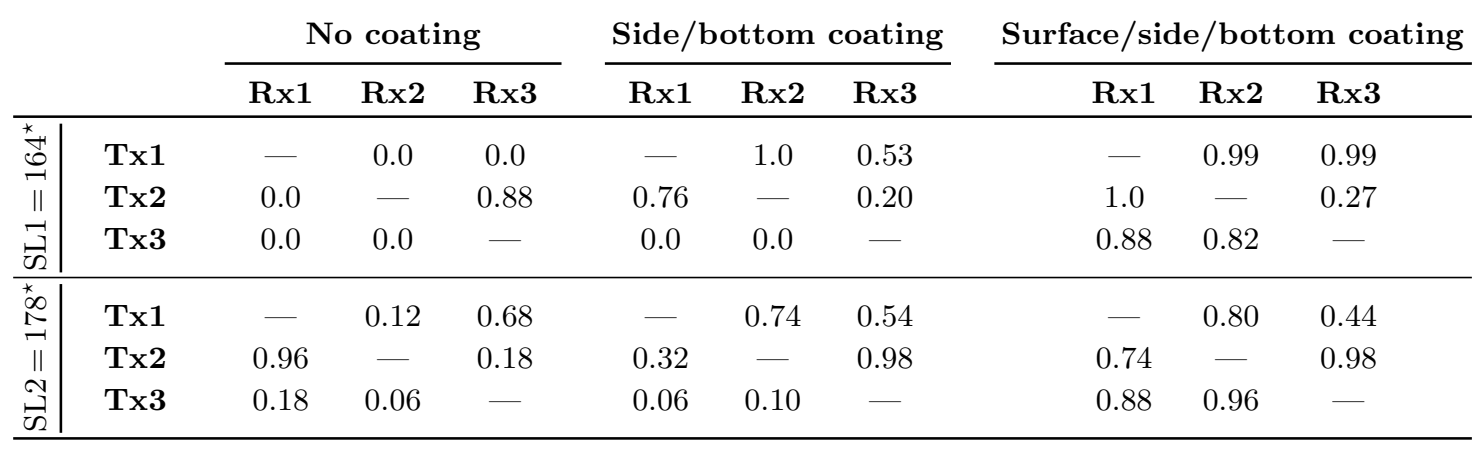

* $\mathrm{SL}$ values are expressed in $\mathrm{dB}$ re $1 \mu \mathrm{Pa}$ at $1 \mathrm{~m}$ from the source.

\begin{abstract}
1008192
1092163

1208105

1316113

1432153

1724143

$2240 \quad 119$

2580220
\end{abstract}

(a)

$\begin{array}{ll}1000 & 415 \\ 1372 & 140 \\ 2612 & 137 \\ 3688 & 150 \\ 4208 & 139 \\ 0 & 0 \\ 0 & 0 \\ 0 & 0\end{array}$

(b)

$\begin{array}{ll}1004 & 422 \\ 0 & 0 \\ 0 & 0 \\ 0 & 0 \\ 0 & 0 \\ 0 & 0 \\ 0 & 0 \\ 0 & 0\end{array}$

(c)
Figure 5: Examples of multipath footprint returned by the firmware of the EvoLogics modem before (a) and after (b), (c) the installation of a phonoabsorbing coating on the walls and surface (Fig. 3c).

ble 3 for the six links between the three modems depicted in Fig. 4, for both SL values considered in this paper and for three tank states: without any coating; after the application of the neoprene coating to the walls and bottom; after the addition of the further neoprene layer on the surface. The tank devoid of any phono-absorbing coating (see also Fig. 2) exhibits very poor performance: by denoting as $i \rightarrow j$ the link from transmitter node $i$ to receiver node $j$, only the link $2 \rightarrow 3$ has a non-zero PDR when the source level is set to SL1. Increasing the source level to SL2 yields generally better performance, where this time links $1 \rightarrow 3$ and $2 \rightarrow 1$ achieve a reasonably high PDR. For all other links, the PDR is significantly lower, and insufficient to ensure stable links for networking experiments.

Adding the neoprene rubber coating to the tank walls and bottom (Fig. 3b) improves the performance of the links for both values of the source level. For SL1, three links $(1 \rightarrow 2$, $1 \rightarrow 3,2 \rightarrow 1$ ) now exhibit reasonably high PDR figures, although the remaining links are still characterized by an exceedingly small PDR. Note that the PDR of link $2 \rightarrow 3$ is now lower due to the slight displacement of the nodes after the application of the phono-absorbing coating. With SL2, the situation improves slightly, as all links achieve a non-zero PDR, although $3 \rightarrow 1$ and $3 \rightarrow 2$ are still quite unreliable. Also, some PDR values appear lower than with SL1, most likely due to the occasional saturation of the receiver input. In any event, this configuration could already be employed to emulate a network where node 3 is the sink (as both nodes 1 and 2 have a good link to it) and occasional communications among nodes 1 and 2 can occur over an asymmetric link.

The best performance, however, is achieved by applying the neoprene coating to the top of the tank, submerged a few mm below the water surface (see Fig.3c). In this case, all links exhibit fairly good performance for both SL1 and SL2. In particular, with the lower source level SL1, the phonoabsorbing surfaces tend to suppress the majority of the secondary paths: this translates into high PDR for almost all links, and in an improvement of $2 \rightarrow 3$ with respect to the case with only side/bottom coating. On the contrary, with SL2 the multipath appears to have been sufficiently mitigated, but we still suspect that the high source level tends to cause input saturation at the receiver, which explains the imperfect PDR figures for some links.

We now zoom in on the measured multipath footprint in the presence of different amounts of phono-absorbing coating, as provided by the firmware of the EvoLogics modem. The modulation scheme implemented on the modems enables the separation of multipath arrivals characterized by different delays, which appear at the receiver as signal components at different frequencies. The modem measures the integrity ${ }^{1}$ of each arrival, and returns a list of the measured delay and integrity of the up to eight strongest arrivals.

Fig. 5 shows an example of multipath recorded by the modem. Each pair of columns displays the time of arrival in ms (left, which would align with the propagation delay of the arrival if the transmitter and receiver were synchronized) and the integrity (right) of each recorded arrival. Fig. 5a refers to a transmission carried out before applying any phonoabsorbing material, whereas Figs. $5 \mathrm{~b}$ and $5 \mathrm{c}$ represent two outcomes of a packet transmission in a fully-coated tank. We clearly notice a strong reduction of the multipath for the second and third transmission, whereby not only the number of recorded paths is less than eight, but there also exists a clearly distinguishable arrival, which is sometimes the only one (as in Fig. 5c). We also note that the first path in Figs. 5b and 5c is not as apparent in Fig. 5a. This is probably due to the negative superposition between secondary paths and the direct path. Secondary paths have a much more limited effect after applying the coating.

However, this is not the only impact of the phono-absorbing coating. Being able to reduce the energy of multipath arrivals with respect to the line-of-sight arrival has also the effect to make integrity values more stable over time, and translates into more reliable links. In order to demonstrate

\footnotetext{
${ }^{1}$ The integrity is a function of the peak value of the crosscorrelation between the received physical layer preamble of each arrival and the preamble template, and can achieve a maximum value of 512 .
} 


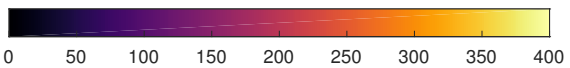

TX 1 RX 2

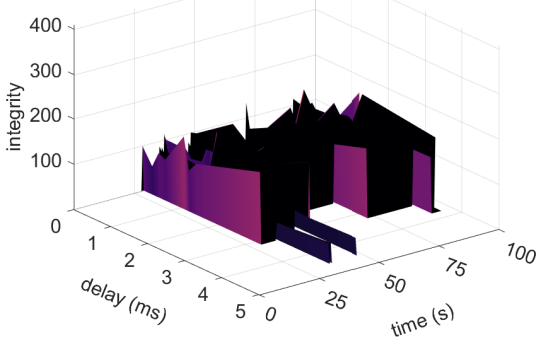

(a)

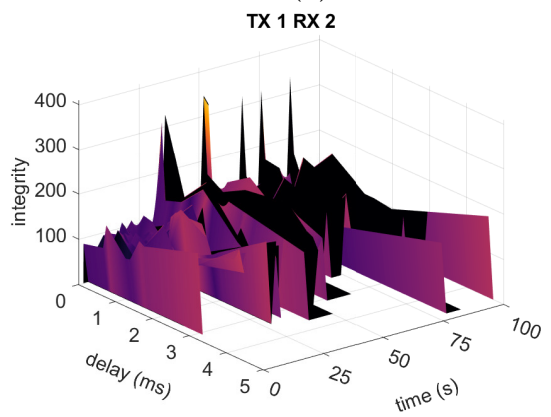

(d)

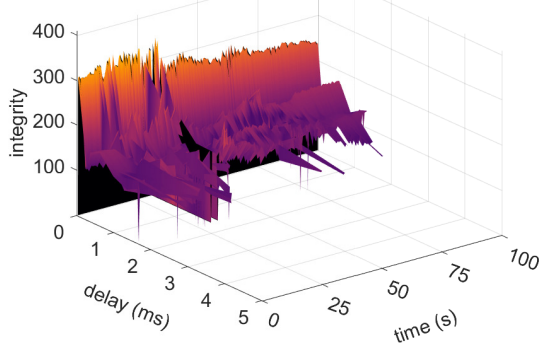

(b)

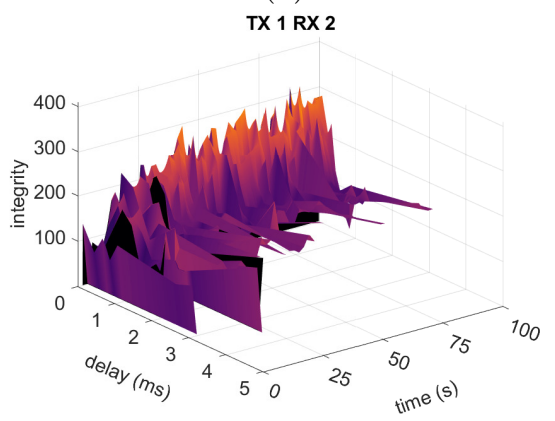

(e)

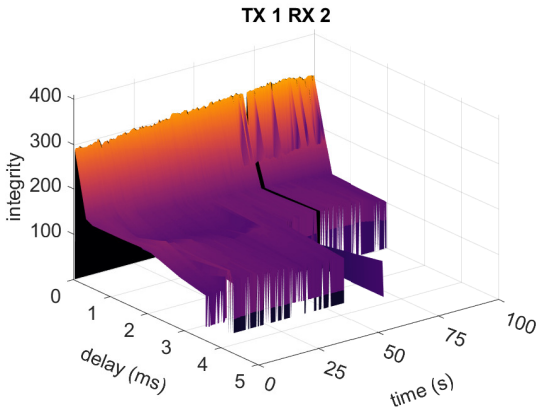

(c)

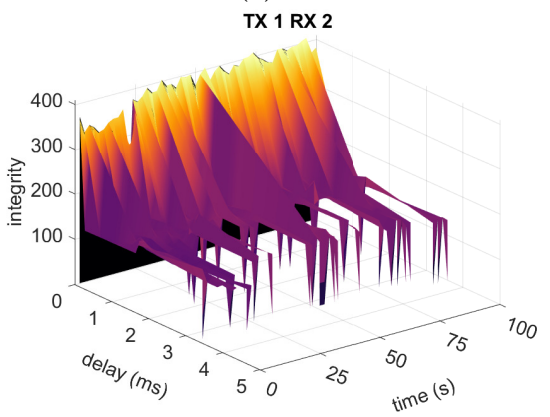

(f)

Figure 6: Evolution of the integrity of multipath arrivals over time as measured by the firmware of the EvoLogics modem for link $1 \rightarrow 2$. Panels (a)-(c) refer to SL1; panels (d)-(f) refer to SL2. Left panels: uncoated tank; center panels: bottom and wall coating; right panels: full coating.

this, we show in Fig. 6 the evolution of the integrity measured by node 2 when it receives packets from node 1 . The first row of three figures is obtained by setting the source level SL1, whereas the second row refers to SL2. In both cases, we measured the integrity of the multipath arrivals in the absence of phono-absorbing coating (Figs. 6a and 6d), in the presence of bottom and wall coating (Figs. 6b and 6e), as well as with a fully coated tank (Figs. 6c and 6f). Each figure depicts the three-dimensional plot of the integrity of each path over time as a function of the arrival delay of that path, referred to the delay of the first arrival. One multipath measurement is taken for each transmitted packet: these snapshots are reported sequentially as a function of the time epoch when the transmission was carried out.

We start from Fig 6a, where we notice that no clearly prominent path exists, and in any event no path achieves a significant integrity. ${ }^{2}$ In this case, it is almost impossible for the receiver to lock onto a clean signal, and in fact no correct reception is obtained in our experiment (see also Table 3). The application of the coating to the bottom and walls of the tank (Fig. 6b) already improves this situation considerably. Now a clear line-of-sight path with integrity above 200 exists; however, its quality oscillates over time, and multipath mitigation is still imperfect, as we still detect a significant component (corresponding to the surface reflection) that achieve an integrity of slightly less than 200 . The quality of the link improves even more after the application of the surface layer (Fig. 6c), which considerably attenuates

\footnotetext{
${ }^{2}$ A rule-of-thumb for achieving a correct reception with the EvoLogics modem is that at least one multipath component exists with an integrity greater than or equal to 200 [6].
}

the surface reflection, and leaves a clean direct path with integrity between 250 and 300, with minor secondary reflections reaching an integrity of about 100 or less.

When we switch the source level to SL2, we observe a slight improvement in the absence of phono-absorbing coating (Fig. 6d), as in a few cases there exists a clear integrity peak corresponding to a clean signal that the receiver can acquire. This reflects the small but non-zero PDR equal to 0.12 reported in Table 3 . When the neoprene coating is applied to the walls and bottom (Fig. 6e), we observe a situation similar to the case of SL1, except that the integrity of the line-of-sight path is slightly lower on average. This and the presence of occasionally significant reflections on the water surface concur to a PDR equal to 0.74 , lower than achieved in the case of SL1. In fact, the application of the surface layer (Fig. 6f) mitigates multipath considerably. The higher source level than SL1 provides a clean (albeit oscillating) direct path with an integrity mostly above 300 , and occasional secondary paths with integrity 100 or lower, several ms later than the line-of-sight path.

We finally emulate a near-far scenario in the tank setup of Fig. 4 by wrapping a layer of neoprene rubber around node 2 . This makes link $1 \rightarrow 3$ strong and link $2 \rightarrow 3$ weaker but still usable. We then have node 1 transmit 100 packets sequentially, and node 2 transmit 50 packets superimposed to the last 50 of node 1 . The test showed that link $1 \rightarrow 3$ still works despite the interference from node 2 , whereas reception stops if the neoprene wrap is removed. Moreover, as expected, increasing the transmit power of node 2 with respect to that of node 1 makes node 3 receive only from node 2 when it transmits together with node 1 , as expected. 


\section{CONCLUSIONS}

In this paper, we presented a detailed survey of phonoabsorbing materials suitable to be employed for multipath mitigation in a small-sized and low-cost HDPE tank for preliminary in-lab experimentation of underwater networking protocols and systems. After a balance of pros and cons, we chose to employ neoprene rubber for its good echo reduction and internal absorption properties. We measured the performance of the coating in terms of the PDR achieved by underwater acoustic modems placed submerged in the tank in three cases: no coating; tank walls and bottom coating; full coating, including a subsurface layer.

From our experimental results, we can conclude that the neoprene rubber serves well the purpose of mitigating multipath inside the tank, and considerably increases the reliability of the acoustic links. We believe this makes our solution a good low-cost alternative to larger and more expensive tanks or pools, which would require a sizeable infrastructural investment. Future work includes mitigating the echo originating at the interface between the outer walls of the HDPE tank and air.

\section{REFERENCES}

[1] S. K. Dhurandher, M. S. Obaidat, and M. Gupta, "An acoustic communication based AQUA-GLOMO simulator for underwater networks," Springer Human-centric Computing and Information Sciences, vol. 2, no. 3, 2012. [Online]. Available: http://dx.doi.org/10.1186/2192-1962-2-3

[2] P. Casari et al., "Open-source suites for the underwater networking community: WOSS and DESERT Underwater," IEEE Network, special issue on "Open Source for Networking: Development and Experimentation", vol. 28, no. 5, pp. 38-46, Sep. 2014.

[3] Y. Zhu, S. Le, L. Pu, X. Lu, Z. Peng, J.-H. Cui, and M. Zuba, "Aqua-Net Mate: A real-time virtual channel/modem simulator for Aqua-Net," in Proc. MTS/IEEE Oceans, Bergen, Norway, Jun. 2013.

[4] M. Chitre, R. Bhatnagar, and W. S. Soh, "UnetStack: An agent-based software stack and simulator for underwater networks," in Proc. MTS/IEEE OCEANS, St. John's, NL, Canada, Sep. 2014.

[5] C. Petrioli, R. Petroccia, J. R. Potter, and D. Spaccini, "The SUNSET framework for simulation, emulation and at-sea testing of underwater wireless sensor networks," Elsevier Ad Hoc Networks, S.I. Advances in Underwater Commun. and Networks, vol. 34, pp. 224-238, Nov. 2015.

[6] EvoLogics, "S2C modem quick test guide," Sep. 2013.

[7] "CMRE facilities and equipment," Last time accessed: June 2016. [Online]. Available: http://www.cmre.nato.int/research/facilities-a-equipment

[8] G. Toso, I. Calabrese, P. Casari, and M. Zorzi, "RECORDS: a remote control framework for underwater networks," in Proc. IEEE/IFIP Med-Hoc-Net, Piran, Slovenia, Jun. 2014.

[9] S. Shankar and M. Chitre, "Tuning an underwater communication link," in Proc. MTS/IEEE OCEANS, Bergen, Norway, Jun. 2013.

[10] E. Demirors, G. Sklivanitis, T. Melodia, S. Batalama, and D. Pados, "Software-defined underwater acoustic networks: toward a high-rate real-time reconfigurable modem," IEEE Commun. Mag., vol. 53, no. 11, pp. 64-71, Nov. 2015.

[11] R. Odugoudar and I. Nissen, "Ad-hoc network emulation framework for underwater communication applications," in Proc. ACM WUWNet, Woods Hole, MA, Sep. 2010.
[12] "S2CR 18/34 Acoustic Modem," Last time accessed: June 2016. [Online]. Available: http://www.evologics.de/en/ products/acoustics/s2cr_18_34.html/

[13] R. Diamant, P. Casari, F. Campagnaro, and M. Zorzi, "A handshake-based protocol exploiting the near-far effect in underwater acoustic networks," IEEE Wireless Commun. Lett., vol. 5, no. 3, pp. 308-311, Jun. 2016.

[14] L. Bjørnø and M. Kjeldgaard, "A wide frequency band anechoic water tank," Acta Acustica united with Acustica, vol. 32, no. 2, pp. 103-109, Feb. 1975.

[15] E. Meyer, "Construction of an anechoic underwater sound measuring tank," Navy Underwater Sound Laboratory, Tech. Rep. AD-758 095, Jan. 1965. [Online]. Available: http://www.dtic.mil/dtic/tr/fulltext/u2/758095.pdf

[16] C. L. Darner, "An underwater sound absorber for an anechoic tank," Underwater Sound Reference Lab., Tech. Rep. 31, Sep. 1953. [Online]. Available: https://archive.org/details/DTIC_AD0036133

[17] F. A. Barge, "Underwater acoustic absorption characteristics of composites of wood, rubber, and steel," Naval Sea Systems Command, Tech. Rep. TM 78-52, May 1978. [Online]. Available: http://www.dtic.mil/dtic/tr/fulltext/u2/a059328.pdf

[18] S. Ogata, T. Tanaka, K. Nagakura, H. Nakagawa, and K. Takashima, "The experimental investigation on the sound absorption coefficient of the sound absorptive materials in scale model experiment for railway noise," in Proc. Inter-Noise, Nice, France, Aug. 2000.

[19] J. Zhang and Z. Xue, "Study on under-water sound absorption properties of eucommia ulmoides gum and its blends," Polymer Bulletin, vol. 67, no. 3, pp. 511-525, Jul. 2011.

[20] P. K. Garul and T. K. Chaki, "Acoustic and mechanical properties of neoprene rubber for encapsulation of underwater transducers," Intl. J. of Scientific Engineering and Technology, vol. 1, no. 5, pp. 231-237, Nov. 2012.

[21] Z. Liu and M. Sheng, "Study on characteristics of sound absorption of underwater visco-elastic coated compound structures," Modern Applied Science, vol. 3, no. 1, pp. 32-41, Jan. 2009.

[22] H. Jiang, Y.-R. Wang, M.-L. Zhang, Y.-P. Hu, D. Lan, Q.-L. Wu, and H.-T. Lu, "Wide-band underwater acoustic absorption based on locally resonant unit and interpenetrating network structure," Chinese Physics B, vol. 19, no. 2, pp. 026 202-1-6, Feb. 2010.

[23] Acoustic Polymers Ltd., "Kilohertz acoustic tile," last time accessed: July 2016. [Online]. Available: http://www. acousticpolymers.co.uk/pages/kilohertz-acoustic-tile.html

[24] A. Dobrucki, B. Żółtogórski, P. Pruchnicki, and R. Bolejko, "Sound-absorbing and insulating enclosures for ultrasonic range," Archives of acoustics, vol. 35, no. 2, pp. 157-164, May 2010.

[25] T. Ramotowski and K. Jenne, "NUWC XP-1 polyurethane-urea: a new, "acoustically transparent" encapsulant for underwater transducers and hydrophones," in Proc. MTS/IEEE OCEANS, San Diego, CA, Sep. 2003.

[26] R. N. Capps, "Elastomeric materials for acustical applications," Underwater Sound Ref. Detach. Naval Research Lab., Tech. Rep. A216 872, Sep. 1989. [Online]. Available: http://www.dtic.mil/dtic/tr/fulltext/u2/a059328.pdf

[27] Y. Wang, H. Jiang, M. Chen, and Y. Feng, "A wide band underwater strong acoustic absorbing materials," in Proc. ICEM, Porto, Portugal, Jul. 2012.

[28] Intl. Inst. of Synthetic Rubber Producers, "Synthetic rubber," last time accessed: July 2016. [Online]. Available: http://iisrp.com/site/synthetic-rubber

[29] Precision Acoustics, "Apltile SF5048," last time accessed: July 2016. [Online]. Available: http://www.acoustics.co.uk/product/apltile-sf5048/ 